\title{
Protein aggregate-ligand binding assays based on microfluidic diffusional separation
}

\author{
Yingbo Zhang, ${ }^{\dagger, \dagger}$ Alexander K. Buell, $,+,, \mathbb{I}, \ddagger$ Thomas Müller,,${ }^{\S}$, Erwin De Genst, ${ }^{\dagger, \|}$ \\ Justin Benesch, ${ }^{\perp}$ Christopher M. Dobson, ${ }^{\dagger}$ and Tuomas P. J. Knowles ${ }^{* \dagger}$ \\ Department of Chemistry, University of Cambridge, Lensfield Road, Cambridge CB2 IEW, UK, \\ These authors contributed equally to the work, Current address: Institute of Physical Biology, \\ University of Düsseldorf, Universitätsstr. 1, 40225 Düsseldorf, Germany, Fluidic Analytics Ltd, \\ Unit 5 Chesterton Mill, French's Road, Cambridge, CB4 3NP, UK, Current address: \\ AstraZeneca, Innovative Medicines, Discovery Sciences, Cambridge Science Park, Milton Road, \\ Cambridge CB4 OWG, UK, and Department of Chemistry, Physical and Theoretical Chemistry \\ Laboratory, University of Oxford, South Parks Road, Oxford OX3 1QZ, UK \\ E-mail: alexander.buell@uni-duesseldorf.de; tpjk2@cam.ac.uk
}

\begin{abstract}
The measurement of molecular interactions with pathological protein aggregates, including amyloid fibrils, is of central importance in the context of the development of diagnostic

\footnotetext{
${ }^{*}$ To whom correspondence should be addressed

'Department of Chemistry, University of Cambridge, Lensfield Road, Cambridge CB2 1EW, UK

These authors contributed equally to the work

${ }^{\text {II }}$ Current address: Institute of Physical Biology, University of Düsseldorf, Universitätsstr. 1, 40225 Düsseldorf, Germany

${ }^{\S}$ Fluidic Analytics Ltd, Unit 5 Chesterton Mill, French's Road, Cambridge, CB4 3NP, UK

\|Current address: AstraZeneca, Innovative Medicines, Discovery Sciences, Cambridge Science Park, Milton Road, Cambridge CB4 0WG, UK

${ }^{\perp}$ Department of Chemistry, Physical and Theoretical Chemistry Laboratory, University of Oxford, South Parks Road, Oxford OX3 1QZ, UK
} 
and therapeutic strategies against protein misfolding disorders. Probing such interactions using conventional methods can, however, be challenging due to the supra-molecular nature of protein aggregates, their heterogeneity, and their often dynamic nature. Here we demonstrate that direct measurements of diffusivity using a microfluidic platform, enable both the affinity and kinetics of ligand binding to amyloid fibrils to be determined in the solution state. This method yields rapid binding information while requiring only microlitres of sample, and is therefore a powerful technique for identifying and characterising molecular species with potential therapeutic or diagnostic interest.

\section{Introduction}

Amyloid fibrils are a class of insoluble protein nanostructures rich in $\beta$-sheet secondary structure, ${ }^{1}$ that were initially identified as a result of their involvement in a wide range of protein misfolding disorders such as Alzheimer's and Parkinson's diseases. ${ }^{2,3}$ The binding of a variety of ligands including small molecules ${ }^{4}$ and proteins ${ }^{5}$ to such species is of great importance in the context of understanding and controlling protein aggregation. For example dye molecules, notably Thioflavin-T (ThT), ${ }^{6}$ are used for the quantitative analysis of the time courses of aggregation reactions. Furthermore, small molecules able to interact with amyloid deposits are increasingly powerful as potential diagnostics of neurodegenerative disorders. ${ }^{7}$ In addition, small molecules, peptides and proteins that are able to bind to different molecular species on the aggregation pathway might be able to selectively stabilise certain species and hence influence, or even inhibit the aggregation process. ${ }^{8,9}$ In a recent, innovative application, the high affinity of amyloid aggregates for heavy metal ions has been exploited to remove such species from contaminated water. ${ }^{10}$ Traditional methods to study ligand binding to protein aggregates include isothermal titration calorimetry (ITC) ${ }^{11,12}$ and microdialysis ${ }^{13}$ but these techniques are usually time-consuming and have relatively large sample requirements. Here we describe a microfluidic approach to studying ligand binding to supramolecular structures; microfluidic devices enable precise control and manipulation of smaller volumes (nL) of liquid with higher efficiency than bulk methods. ${ }^{14-18}$ We first demonstrate its ability to 
separate colloid particles of different sizes, and then apply it to study the binding of both small molecules and proteins to amyloid fibrils, as well as in aggregation experiments.

\section{Results and discussion}

\section{Microfluidic diffusional separation device}

A microfluidic device was designed and fabricated in PDMS using soft lithography. ${ }^{19}$ It contains two inlets and two outlets connected by the main channel, in which diffusive separation is able to occur (Figure $1 \mathrm{a}$ and $\mathrm{b}$ ). The length of the input and output channels can be adjusted to generate different hydrodynamic resistances and consequently different flow rates and to generate a flow profile that is sufficiently sharp for the separation of small (sub- $\mu \mathrm{m}$ ) particles, a flow focusing nozzle ten times wider than the main channel was designed and incorporated into the device (Figure $1 \mathrm{c}$ ). ${ }^{18}$ The latter serves to rapidly establish steady-state laminar flow and to decrease the ratio of the diffusion length relative to the whole stream width to obtain a focused steady sample stream in the centre of the main channel and a sharp interface in the nozzle region. The width of the separation outlet was also designed to be adjustable to generate different separation ratios. Due to the well-defined, narrow width of the starting distribution of species, ${ }^{18}$ this diffusion separation device represents an advancement compared to conventional T-sensors or H-filters. ${ }^{15,20}$

In a first set of experiments designed to demonstrate the ability of this device to separate a mixture according to particle size, a solution containing large $(\mathrm{d}=1 \mu \mathrm{m}, \mathrm{red})$ and small $(\mathrm{d}=50 \mathrm{~nm}$, green $)$ fluorescent colloid particles was injected into the central inlet and water was introduced from the side inlets. The two types of colloid particles were selected to have different emission wavelengths enabling them to be observed with a fluorescence microscope through different optical filters. As shown in Figure $1 \mathrm{c}$, the majority of the large colloid particles remained in the centre of the main channel and left through the central outlet, while the small colloids diffused across the entire width of the main channel and reached all three outlets. By adjustment of the flow rate, it was possible to achieve a situation where less than $1 \%$ of the large particles reached the side channels, as measured 
by fluorescence intensity (Figure $1 \mathrm{c}$ ).

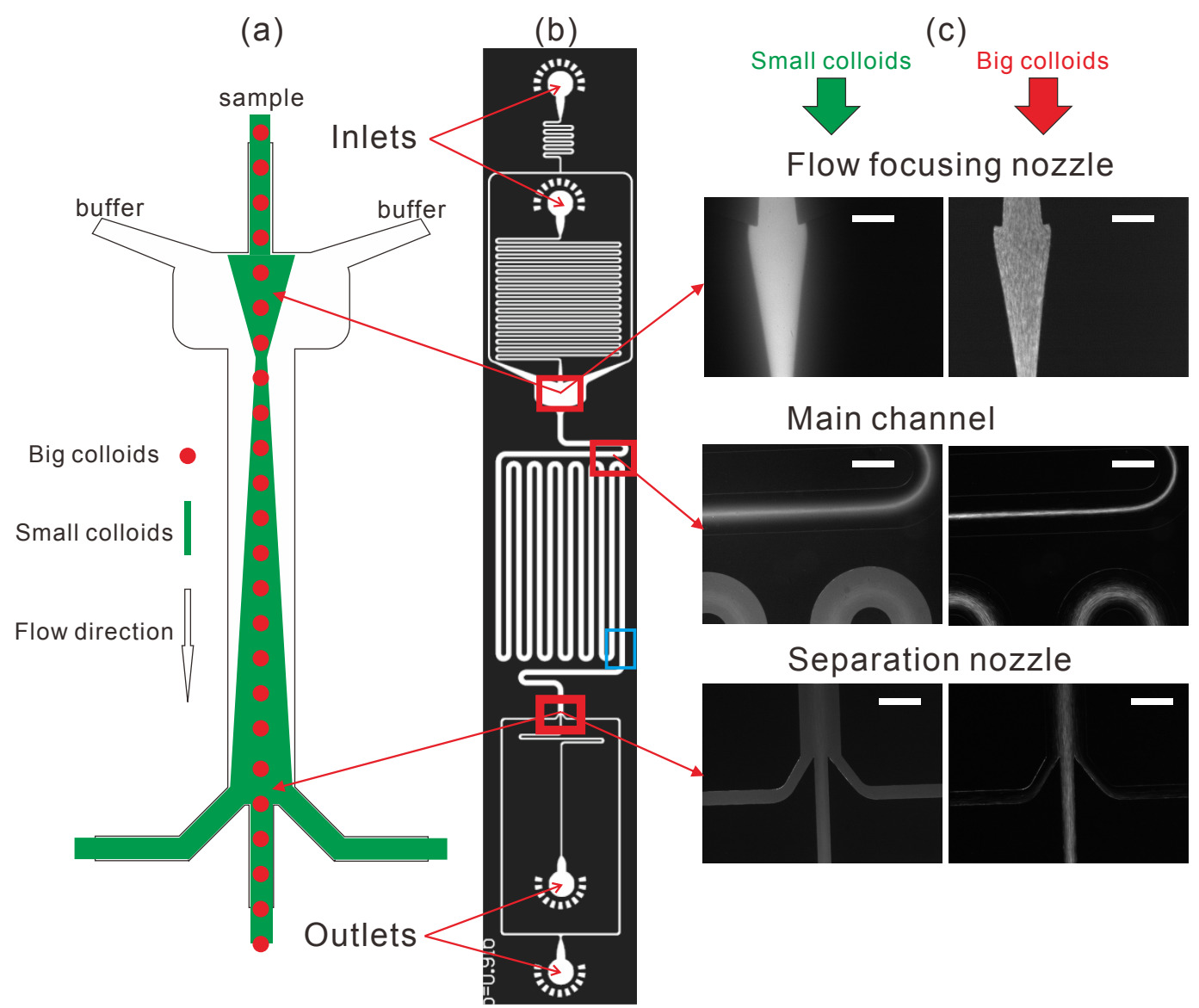

Figure 1: Separation of large $(\mathrm{d}=1 \mu \mathrm{m})$ and small colloidal particles $(\mathrm{d}=50 \mathrm{~nm})$ to characterise the microfluidic device described in this paper. a) Schematic diagram of the separation process. b) Photolithography mask of the microfluidic device. c) Flow profiles of the fluorescent colloid particles at the flow focusing nozzle, main channel and separation nozzle, observed through a green filter (left column, small colloids) or a red filter (right column, large particles). The blue square corresponds to the position where images e)-h) of Figure 4 were taken. Scale bars $=300$ $\mu m$.

\section{Interactions of ThT molecules with insulin fibrils}

Based on the efficient separation of particles of different sizes by the microfluidic device, the binding properties of ligands of protein aggregates can be studied. The fluorescent dye ThT is a commonly used molecular probe of the presence of amyloid structures ${ }^{6}$ as its excitation and emission maxima, as well as its fluorescence quantum yield change strongly when binding to 
amyloid fibrils. ${ }^{21-23}$ A beam containing a mixture of insulin fibrils and ThT molecules (in aqueous solution at $\mathrm{pH}=1.92$ ) was introduced from the central inlet channel, and a solution at the same $\mathrm{pH}$ but without either type of species was introduced from the side inlet channels. As shown in Figure 2, the small ThT molecules diffused and dispersed throughout the main channel and were distributed equally across all the outlet channels, while the insulin fibrils diffused slowly and entered only the central outlet channel. In a control experiment, a solution of ThT at the same concentration and $\mathrm{pH}$, but without insulin fibrils was introduced. In both cases, samples were then collected from the side outlet channels for measurement of the ThT concentration. The experiments were then repeated at a range of different ThT concentrations in order to calculate the binding affinity and stoichiometry.

We used absorption spectroscopy rather than fluorescence intensity measurements in order to quantify the ThT concentrations. The fluorescence of free ThT shows a complex concentration dependence, which is mostly due to the so-called inner filter effect. ${ }^{24}$ The optical densities (absorbances) of ThT solutions, however, correlate closely with their concentrations under all the conditions used in this study. As shown in Figure $2 \mathrm{f}$ ), a well-defined plot of the ratio of the concentration of bound ThT to that of insulin (bound ThT/insulin) against the concentration of free ThT indicates a $K_{d}$ value of $20 \mu \mathrm{M}$ and a stoichiometry $n$ is around 0.11 mole ThT molecules per mole of insulin fibrils, similar to what has been reported under comparable conditions ${ }^{11}$ (see supplementary materials for details of the analysis).

\section{Binding of nanobodies to $\alpha$-synuclein fibrils}

Nanobodies are single-domain antibodies derived from naturally occurring camelid heavy-chain antibodies. Nanobodies are highly stable and soluble and can be raised against a wide range of molecular species, including monomeric, oligomeric and fibrillar protein, and bind to their antigens with high affinity ( $100 \mathrm{nM}-\mathrm{pM}) . .{ }^{5,25,26}$ We used our diffusion separation method to demonstrate and investigate the binding between amyloid fibrils formed from $\alpha$-synuclein, ${ }^{27}$ an intrinsically disordered protein whose aggregation is associated with Parkinson's disease, and the nanobody 


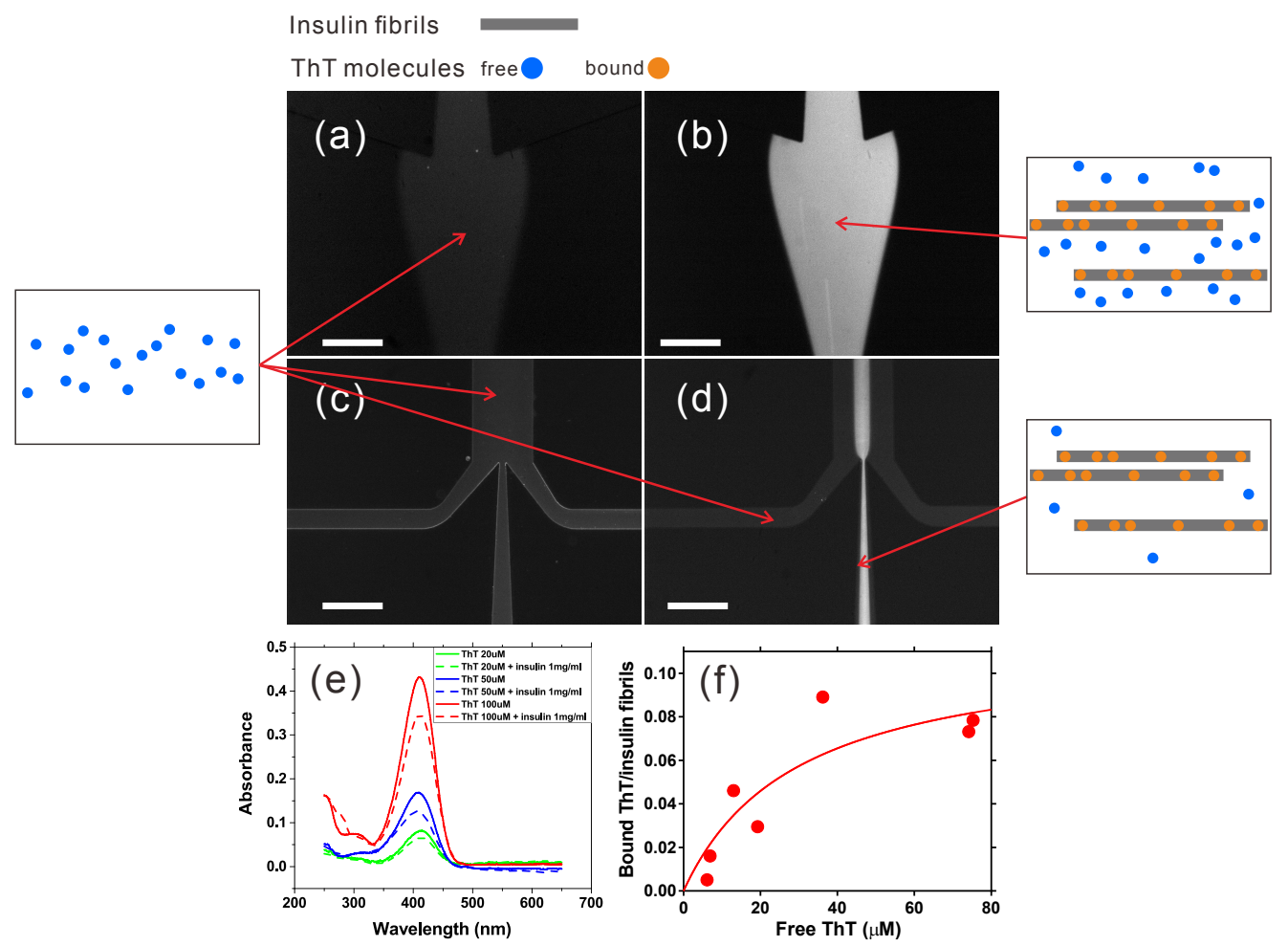

Figure 2: Flow profiles of ThT molecules in the presence and absence of insulin fibrils. a) A ThT solution at the flow focusing nozzle; b) ThT solution containing also insulin fibrils at the flow focusing nozzle; c) ThT solution at the separation nozzle; d) ThT solution containing also insulin fibrils at the separation nozzle. (Blue filter in dark field, Scale bars $=300 \mu \mathrm{m}$ ). e) Absorbance spectra of ThT solutions collected from the side outlets after microfluidic separation of ThT solutions with and without insulin fibrils f) Binding curve for the interaction of ThT molecules with insulin fibrils. 
NbSyn87. ${ }^{5}$ Fluorescence measurements of fibrils of $\alpha$-synuclein (labelled with Alexa $568^{28}$ ) and NbSyn87 (labelled with Alexa 647) were taken using a microscope fitted with a green or a red filter. Experiments with $\alpha$-synuclein fibrils alone showed that they did not diffuse far from the channel centre (Figure 3 a), as expected from their length of several hundred nanometers, ${ }^{27}$ while the monomeric nanobodies were observed to diffuse throughout the whole channel (Figure $3 \mathrm{c}$ ). In contrast, experiments with a mixture of $\alpha$-synuclein fibrils and NbSyn87 (5:1 molar ratio), showed that both the fibrils and the nanobodies, selectively monitored through the green and red filters, remained in the channel centre (Figure $3 \mathrm{~b}, \mathrm{~d}$ ), indicating that the nanobodies were strongly bound to the $\alpha$-synuclein fibrils.

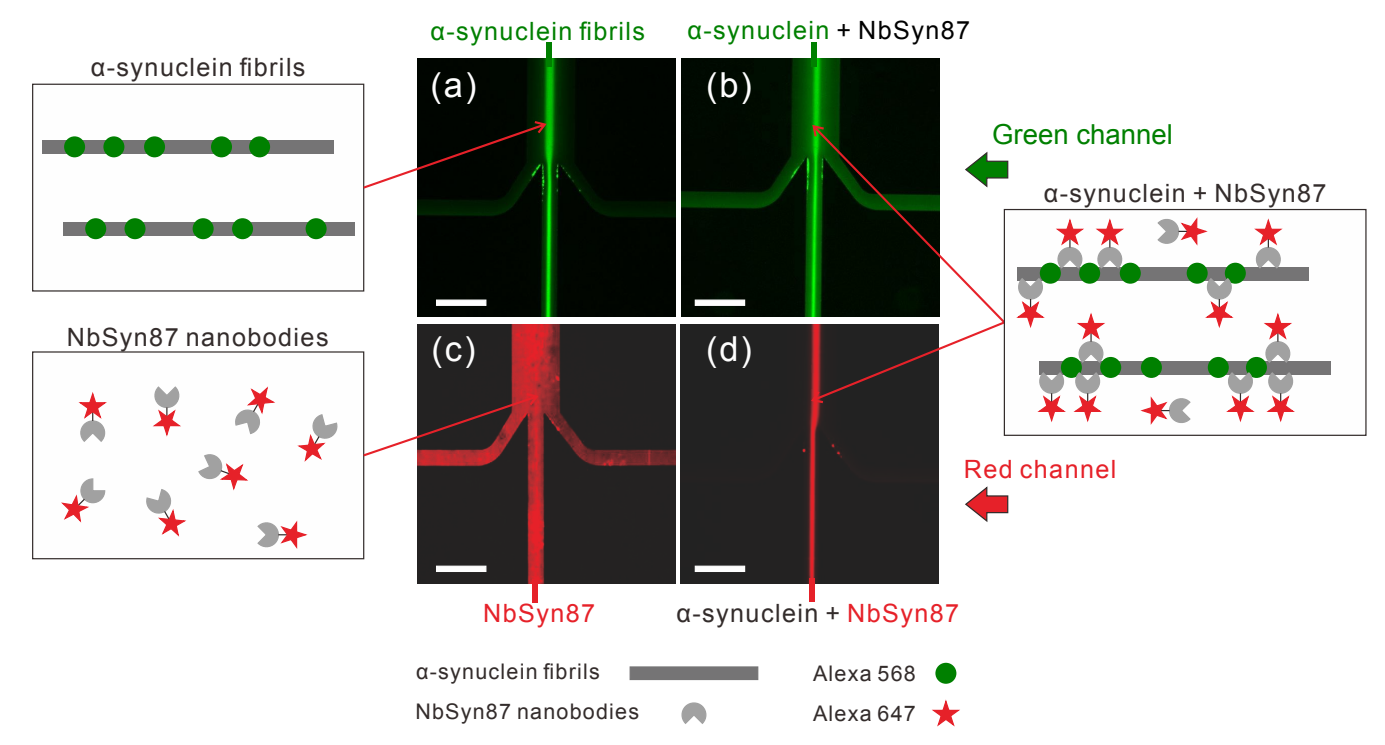

Figure 3: Fluorescence measurements of NbSyn87 binding to $\alpha$-synuclein fibrils after diffusional separation. a) pure $\alpha$-synuclein fibrils observed through a green filter; b) mixture of $\alpha$-synuclein and NbSyn87, green filter; c) pure NbSyn87 observed through a red filter; d) mixture of $\alpha$ synuclein and NbSyn87, red filter; Scale bars $=300 \mu \mathrm{m}$

\section{Monitoring the growth kinetics of $\alpha$-synuclein fibrils.}

The experimental results in the previous sections demonstrate that the diffusional properties of amyloid fibrils are sufficiently different from those of both small molecules and monomeric macromolecules to achieve efficient separation of those species using the microfluidic device. It should, 
therefore, be possible to separate amyloid fibrils from free monomeric protein, that has not yet reacted with the fibrils. It has been demonstrated in the past that microfluidics can be used to study the kinetics of protein aggregation, ${ }^{29,30}$ but these methods do not exploit the differences in size and hence diffusion coefficient between monomeric and aggregated protein. In Figure 4 a-d we demonstrate with $\alpha$-synuclein that such studies can indeed be carried out rapidly and effectively. We labeled monomeric and fibrillar $\alpha$-synuclein with different colours. ${ }^{28}$ As expected from the results in the previous section, the fibrils remain localised in the centre of the channel, whilst the monomers diffuse across the channel. When a solution containing both monomeric and fibrillar protein species is incubated for different lengths of time $(0,10$ and $40 \mathrm{~min}$, Figure $4 \mathrm{e}-\mathrm{h})$, the fluorescence signal corresponding to the monomeric protein was increasingly localised in the centre of the channel, as a consequence of its incorporation into the aggregates. The fluorescence intensity outside the bright central region (Figure 4 i, averaged along the channel), corresponding to remaining freely diffusing monomer, at the different time points can be fitted to an exponential function (see supplementary material) to determine the elongation rate constant under these conditions. ${ }^{27}$ In addition to thermodynamic binding measurements, our method can therefore be used to determine the overall kinetics of aggregation processes that occur on a minute time scale, while the kinetics of individual binding events are beyond the time resolution of this method.

\section{Conclusions}

We have developed and tested an efficient method for monitoring the binding of small and large molecules to amyloid fibrils based on microfluidic diffusional separation. The method was used successfully to characterise the binding of ThT to insulin fibrils, of single domain antibodies to $\alpha$-synuclein fibrils and also the growth of $\alpha$-synuclein fibrils. The method enables qualitative binding information to be rapidly obtained, while only requiring a few $\mu 1$ of sample. For quantitative studies, a series of measurements under different conditions enables binding constants and stoichiometries to be established. If the molecules under study are fluorescent, data analysis can be 


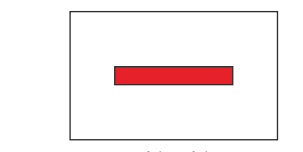

Fibrils
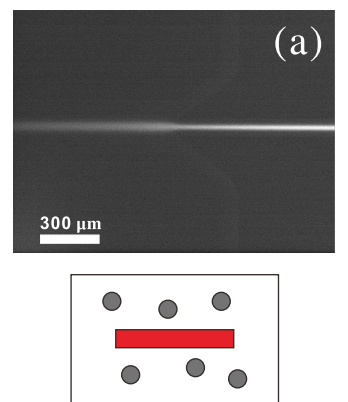

Fibrils+Monomers
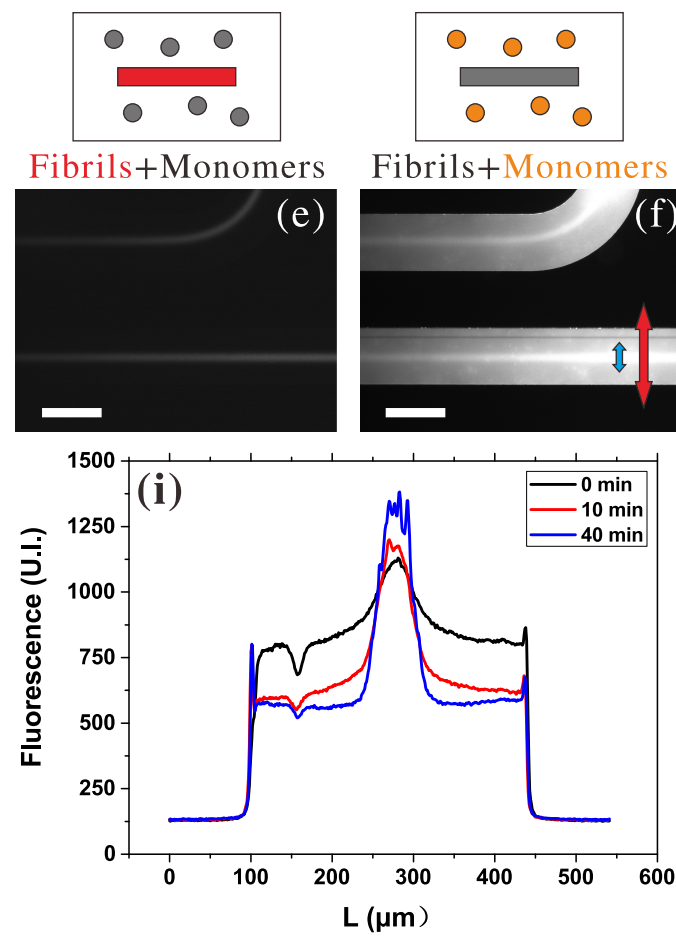

Fibrils+Monomers

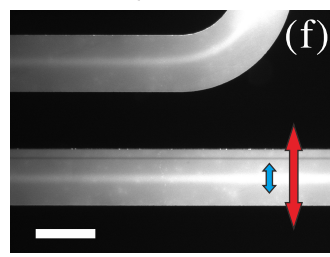

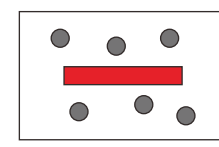

Fibrils+Monomers

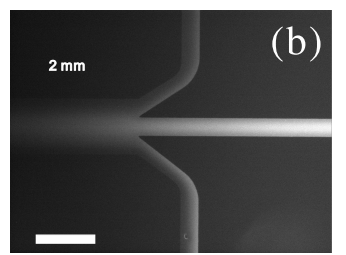

Fibrils+Monomers
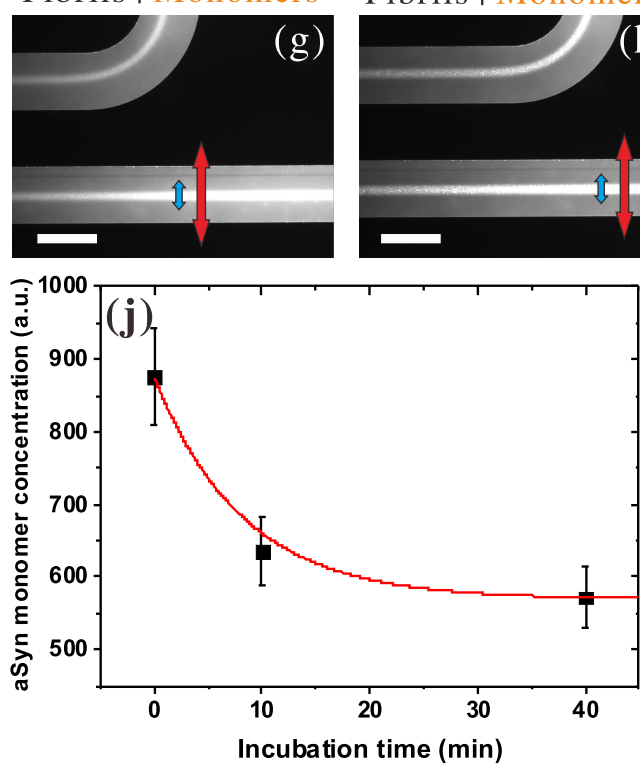

Figure 4: Monitoring the growth of $\alpha$-synuclein fibrils using diffusional separation. a) $\alpha$-synuclein fibrils, fluorescently labelled with Alexa $647 \mathrm{~b}$ ) Monomeric $\alpha$-synuclein, fluorescently labelled with Alexa 568, at $300 \mu \mathrm{l} / \mathrm{h}$ c) Mixture of $\alpha$-synuclein amyloid fibrils (647) and monomeric $\alpha$ synuclein (569), observed through a red filter d) Same as c), but observed through a green filter. Experiments in a)-d) were all performed at withdrawal rate of $300 \mu \mathrm{l} / \mathrm{h} \mathrm{e}$ )-j) Measurement of $\alpha$ synuclein fibril elongation. e)-f) Mixture of fibrils and monomer at the position indicated by the blue square in Figure 1. These experiments were performed at a withdrawal rate of $100 \mu \mathrm{l} / \mathrm{h} \mathrm{g}$ ) after 10 min incubation of the solution containing monomers and fibrils. h) after 40 min incubation. i) fluorescence intensity profiles across the channel of the images in f,g,h, at the positions indicated by the red arrows. j) Decrease of free monomer signal outside the bright central region (indicated by blue arrows) over time during the growth of the fibrils. The data was fitted to an equation of the form $F(t)=F_{0}+A \cdot \exp \left(-t / t_{0}\right)$ (see supplementary material), confirming the exponential decrease of free monomer in seeded aggregation. ${ }^{27}$ 
rapidly performed in situ, at nM concentrations. However, in contrast to other low volume. high throughput technologies for biomolecular binding, such as for example microscale thermophoresis, ${ }^{26}$ sample autofluorescence or fluorescent labeling, the latter being potentially able to interfere with the interaction of interest, e.g. through steric or electrostatic effects, is not a necessary requirement for our method, due to the possibility to collect the sample from the outlet and determine the concentrations by other methods.

This method has applications ranging beyond the study of molecules binding to amyloid fibrils, and should be readily applicable in all cases involving particles that differ by at least one order of magnitude in size and hence in diffusion coefficient. We anticipate that such diffusional separation modules will be useful in many other areas of study, for example the self-assembly of functional nanostructures in microfluidic flow, where the final structures can be separated from the building blocks.

\section{Materials and Methods}

A complete and detailed description of the methods can be found in the supplementary material.

\section{Acknowledgement}

This work was supported by the UK Biotechnology and Biological Sciences Research Council (CMD, TPJK, BB/E019927/1), the Wellcome Trust (CMD, TPJK, 094425/Z/10/Z), The Frances and Augustus Newman Foundation (TPJK), Magdalene College, Cambridge (AKB) and the Leverhulme Trust (AKB, ECF-2013-299). Y.Z. thanks EPSRC and the Cambridge NanoDTC as well as Fluidic Analytics for support. J.L.P.B. is a University Research Fellow of the Royal Society.

\section{References}

(1) Makin, O. S.; Serpell, L. C. FEBS J 2005, 272, 5950-5961. 
(2) Jarrett, J. T.; Lansbury, P. T. Cell 1993, 73, 1055-1058.

(3) Dobson, C. M. Nature 2003, 426, 884-890.

(4) Buell, A. K.; Esbjörner, E. K.; Riss, P. J.; White, D. A.; Aigbirhio, F. I.; Toth, G.; Welland, M. E.; Dobson, C. M.; Knowles, T. P. J. Phys Chem Chem Phys 2011, 13, 2004420052.

(5) Guilliams, T.; El-Turk, F.; Buell, A. K.; O’Day, E. M.; Aprile, F. A.; Esbjörner, E. K.; Vendruscolo, M.; Cremades, N.; Pardon, E.; Wyns, L.; Welland, M. E.; Steyaert, J.; Christodoulou, J.; Dobson, C. M.; Genst, E. D. J Mol Biol 2013, 425, 2397-2411.

(6) LeVine, H. Protein Sci 1993, 2, 404-410.

(7) Klunk, W. E. et al. Ann Neurol 2004, 55, 306-319.

(8) Bulawa, C. E.; Connelly, S.; Devit, M.; Wang, L.; Weigel, C.; Fleming, J. A.; Packman, J.; Powers, E. T.; Wiseman, R. L.; Foss, T. R.; Wilson, I. A.; Kelly, J. W.; Labaudinière, R. Proc Natl Acad Sci U S A 2012, 109, 9629-9634.

(9) Cohen, S. I. A.; Arosio, P.; Presto, J.; Kurudenkandy, F. R.; Biverstål, H.; Dolfe, L.; Dunning, C.; Yang, X.; Frohm, B.; Vendruscolo, M.; Johansson, J.; Dobson, C. M.; Fisahn, A.; Knowles, T. P. J.; Linse, S. Nat Struct Mol Biol 2015, 22, 207-213.

(10) Bolisetty, S.; Mezzenga, R. Nat Nanotechnol 2016, 11, 365-371.

(11) Groenning, M.; Norrman, M.; Flink, J. M.; van de Weert, M.; Bukrinsky, J. T.; Schluckebier, G.; Frokjaer, S. J Struct Biol 2007, 159, 483-497.

(12) Jung, J.-M.; Savin, G.; Pouzot, M.; Schmitt, C.; Mezzenga, R. Biomacromolecules 2008, 9 , $2477-2486$.

(13) Kuznetsova, I. M.; Sulatskaya, A. I.; Uversky, V. N.; Turoverov, K. K. PLoS One 2012, 7, e30724. 
(14) Brody, J. P.; Yager, P. Sensors and Actuators A: Physical 1997, 58(1), 13-18.

(15) Weigl, B. H.; Yager, P. Science 1999, 283, 346-347.

(16) Hatch, A.; Garcia, E.; Yager, P. Proceedings of the IEEE 2004, 92(1), 126-139.

(17) Kamholz, A. E.; Weigl, B. H.; Finlayson, B. A.; Yager, P. Anal. Chem. 1999, 71(23), 53405347.

(18) Arosio, P.; Müller, T.; Rajah, L.; Yates, E. V.; Aprile, F. A.; Zhang, Y.; Cohen, S. I. A.; White, D. A.; Herling, T. W.; Genst, E. J. D.; Linse, S.; Vendruscolo, M.; Dobson, C. M.; Knowles, T. P. J. ACS Nano 2016, 10, 333-341.

(19) Duffy, D. C.; McDonald, J. C.; Schueller, O. J. A.; Whitesides, G. M. Anal. Chem. 1998, 70, 1998, 70, 4974-4984.

(20) Cohen, S. I. A.; Knowles, T. P. J.; Dobson, C. M.; Rajah, L.; White, D. EP2912455 - Fluidic Device. 2014.

(21) Groenning, M. J Chem Biol 2010, 3, 1-18.

(22) Biancalana, M.; Koide, S. Biochim Biophys Acta 2010, 1804, 1405-1412.

(23) Sulatskaya, A. I.; Kuznetsova, I. M.; Turoverov, K. K. J Phys Chem B 2012, 116, 2538-2544.

(24) Fonin, A. V.; Sulatskaya, A. I.; Kuznetsova, I. M.; Turoverov, K. K. PLoS One 2014, 9, e103878.

(25) Genst, E. J. D.; Guilliams, T.; Wellens, J.; O’Day, E. M.; Waudby, C. A.; Meehan, S.; Dumoulin, M.; Hsu, S.-T. D.; Cremades, N.; Verschueren, K. H. G.; Pardon, E.; Wyns, L.; Steyaert, J.; Christodoulou, J.; Dobson, C. M. J Mol Biol 2010, 402, 326-343.

(26) Wolff, M.; Mittag, J. J.; Herling, T. W.; Genst, E. D.; Dobson, C. M.; Knowles, T. P. J.; Braun, D.; Buell, A. K. Sci Rep 2016, 6, 22829. 
(27) Buell, A. K.; Galvagnion, C.; Gaspar, R.; Sparr, E.; Vendruscolo, M.; Knowles, T. P. J.; Linse, S.; Dobson, C. M. Proc Natl Acad Sci U S A 2014, 111(21), 7671-7676.

(28) Pinotsi, D.; Buell, A. K.; Galvagnion, C.; Dobson, C. M.; Schierle, G. S. K.; Kaminski, C. F. Nano Lett 2014, 14, 339-345.

(29) Lee, J. S.; Ryu, J.; Park, C. B. Anal Chem 2009, 81, 2751-2759.

(30) Saar, K.-L.; Yates, E. V.; Müller, T.; Saunier, S.; Dobson, C. M.; Knowles, T. P. J. Biophys J 2016, $110,555-560$. 


\section{Supplementary material: Protein aggregate-ligand}

\section{binding assays based on microfluidic diffusional}

\section{separation}

Yingbo Zhang, ${ }^{\dagger}$ Alexander K. Buell, ${ }^{*, \dagger}, \ddagger, \mathbb{I}$ Thomas Müller $,{ }^{\S},{ }^{\dagger}$ Erwin De Genst, ${ }^{\dagger}, \|$

Justin Benesch, ${ }^{\perp}$ Christopher M. Dobson, ${ }^{\dagger}$ and Tuomas P. J. Knowles ${ }^{* \dagger}$

Department of Chemistry, University of Cambridge, Lensfield Road, Cambridge CB2 1EW, UK, Current address: Institute of Physical Biology, University of Düsseldorf, Universitätsstr. 1, 40225

Düsseldorf, Germany, These authors contributed equally to the work, Fluidic Analytics Ltd,

Cambridge, UK, Current address: AstraZeneca, Innovative Medicines, Discovery Sciences,

Cambridge Science Park, Milton Road, Cambridge CB4 OWG, UK, and Department of Chemistry, Physical and Theoretical Chemistry Laboratory, University of Oxford, South Parks Road, Oxford OX3 1QZ, UK

E-mail: alexander.buell@uni-duesseldorf.de; tpjk2@cam.ac.uk

\footnotetext{
${ }^{*}$ To whom correspondence should be addressed

†Department of Chemistry, University of Cambridge, Lensfield Road, Cambridge CB2 1EW, UK

† Current address: Institute of Physical Biology, University of Düsseldorf, Universitätsstr. 1, 40225 Düsseldorf, Germany

${ }^{\text {II }}$ These authors contributed equally to the work

${ }^{\S}$ Fluidic Analytics Ltd, Cambridge, UK

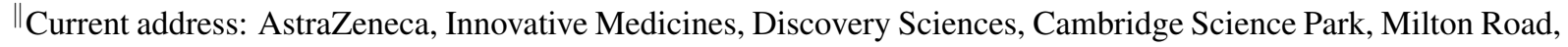
Cambridge CB4 0WG, UK

${ }^{\perp}$ Department of Chemistry, Physical and Theoretical Chemistry Laboratory, University of Oxford, South Parks Road, Oxford OX3 1QZ, UK
} 


\section{Materials and Methods}

Materials The colloids used were Fluoro-Max Fluorescent Polymer Microspheres (Thermo Fisher Scientific, Cat. No. G50 (50 nm) and R0100 (1 $\mu \mathrm{m}))$. Bovine insulin and Thioflavin-T (ThT) were purchased from Sigma-Aldrich (Gillingham, UK). Insulin amyloid fibrils were prepared by stirring a $5 \mathrm{mg} / \mathrm{ml}$ insulin solution in $10 \mathrm{mM} \mathrm{HCl}$ at $60^{\circ} \mathrm{C}$ for $24 \mathrm{~h}$, which corresponds to conditions where the native state of insulin is strongly destabilized and hence fibril formation is rapid. ${ }^{1}$ Human $\alpha$ synuclein was expressed and purified as described previously. ${ }^{2}$ Fluorescent labelling at position 122 was carried out using the N122C variant and Alexa Fluo malimides (Life Technologies Ltd, Paisley, UK), also as described previously. ${ }^{3}$ The production of amyloid fibrils from mixtures of fluorescently labeled and unlabeled protein has also been described previously. ${ }^{3}$ In short, a mixture of ca. $5 \%$ labelled and $95 \%$ unlabelled $\alpha$-synuclein was incubated with a small amount of pre-formed, unlabelled seed fibrils. We have previously shown that the addition of the fluorescent label at position 122 does not interfere with the kinetics of fibril elongation and therefore is a minimally invasive modification. ${ }^{3}$ The antibody NBSyn87 was expressed and purified as described previously ${ }^{4}$ and fluorescently labeled using Alexa Fluo-NHS ester, under conditions where mostly the N-terminal amine is labeled.

Microfluidic device fabrication The fabrication of the diffusional separation devices followed standard soft lithography procedures. ${ }^{5}$ The devices were designed using the AutoCAD software, and a photomask was printed by Micro Lithography Services Ltd (Chelmsford, UK). A layer of SU-8 3025 photoresist (MicroChem Corp, Westborough, MA, USA) was evenly spread on a silicon wafer by spin coating at $3000 \mathrm{rpm}$ (G3 series spin coater, SCS, Indianapolis, USA). After baking at $90^{\circ} \mathrm{C}$ for $12 \mathrm{~min}$, the wafer was covered by the photomask and exposed to UV light for $15 \mathrm{~s}$ with a collimated UV light source (OAI Instruments, San Jose, CA, USA) at $60 \mathrm{~V}$ and $4 \mathrm{~A}$ and baked for another 5 minutes. The photoresist was then developed with propylene glycol methyl ether acetate (PGMEA) and rinsed with isopropyl alcohol (IPA) to produce a positive pattern master.

A 10:1 PDMS/crosslinker (Elastomer 184, Dow Corning, Midland, MI, USA) mixture was poured 
onto the master and degassed under vacuum for $30 \mathrm{~min}$, and then heated to $65^{\circ} \mathrm{C}$ for $2 \mathrm{~h}$. The rigid PDMS was then removed from the master and inlet holes were made with a Harris Uni-Core biopsy punch with $0.75 \mathrm{~mm}$ I.D. (Sigma-Aldrich, Gillingham, UK). Finally the PDMS was plasma bonded to a glass slide (50 mW, $10 \mathrm{~s}$ ) using an Electronic Femto plasma oven (Diener Electronic GmbH, Ebhausen, Germany).

Characterization of binding Fluorescence spectra were recorded using a Cary Eclipse Fluorescence spectrophotometer (Agilent Technologies, Wokingham, UK) and absorption spectra using a plate reader (POLARstar Omega, BMG Labtech, Aylesbury, UK). The fluid flow in the microfluidic device was controlled by neMESYS Syringe Pumps (Cetoni GmbH, Korbußen, Germany) and observed under an Axio Observer inverted fluorescence microscope (Zeiss, Cambridge, UK). Depending on the experiment, samples were injected into the device, or withdrawn from the device, at flow rates ranging from 100 to $500 \mu \mathrm{l} / \mathrm{h}$. In particular, in the experiment designed to study the binding between Thioflavin-T and insulin amyloid fibrils, sample injection was chosen, as it facilitates the quantitative collection of the solution extracted from the side outlets, for subsequent ThT concentration determination.

Fibril elongation measurements To demonstrate the depletion of free monomer as a result of its incorporation into amyloid fibrils, a mixture of Alexa 647 labelled seed fibrils (10 $\mu \mathrm{M}, 5 \%$ labelled protein, 95\% unlabelled protein) and Alexa 568 labelled monomers (10 $\mu \mathrm{M}, 100 \%$ labelled protein) in $10 \mathrm{mM}$ sodium phosphate buffer at $\mathrm{pH} 7.4$ was injected into the diffusion device at 100 $\mu l$ per hour directly after mixing, after $10 \mathrm{~min}$ of incubation at $65^{\circ} \mathrm{C}$ and after $40 \mathrm{~min}$ of incubation at $65^{\circ} \mathrm{C}$. The growth conditions, in particular the low ionic strength, were chosen based on previous results from studies of $\alpha$-synuclein fibril elongation, ${ }^{6}$ as under these conditions, higher order assembly of the fibrils is negligible. The images were analysed by averaging over rectangular areas outside the bright central region which corresponds to the monomer that has elongated the fibrils. Therefore only the freely diffusing monomer, which has not yet incorporated into the seed fibrils, is counted. The error bars shown in figure $4 \mathrm{j}$ reflect differences in the averages from different 
regions of the images, above and below the bright central region. These are likely to stem mostly from inhomogeneities in the illumination. The non-normalised data of the fluorescence intensity as a function of time can be fitted to a function of the form $\mathrm{F}(\mathrm{t})=\mathrm{F}_{0}+\mathrm{A} \cdot \exp \left(-\mathrm{t} / \mathrm{t}_{0}\right)$, yielding the following values of the fit parameters: $\mathrm{F}_{0}=569.7, \mathrm{~A}=305.5$ and $\mathrm{t}_{0}=8.2 \mathrm{~min}=492 \mathrm{~s}$. The exponential decay time $\mathrm{t}_{0}$ can be expressed as $\mathrm{t}_{0}=\frac{1}{P(0) k_{+}}$, with $\mathrm{P}(0)$ the initial number concentration of seed fibrils and $\mathrm{k}_{+}$the elongation rate constant. ${ }^{6} \mathrm{P}(0)$ can be computed from the mass concentration of added seed fibrils, $\mathrm{M}(0)=10 \mu \mathrm{M}$ as $\mathrm{P}(0)=\mathrm{M}(0) / 180,180$ being the average number of monomers per seed fibril prepared with our sonication protocol. ${ }^{6}$ From this analysis, we obtain an elongation rate constant of $\mathrm{k}_{+}=3.7 \cdot 10^{4} \mathrm{M}^{-1} \mathrm{~s}^{-1}$ under those solution conditions $\left(65^{\circ} \mathrm{C}\right)$. This is in good agreement with the value that we have reported previously at $37^{\circ} \mathrm{C}\left(2.2 \cdot 10^{3} \mathrm{M}^{-1} \mathrm{~s}^{-1}\right)$ and the enthalpic barrier of $52.7 \mathrm{~kJ} / \mathrm{mol}$ that determines the temperature dependence of the elongation reaction. ${ }^{6}$

Diffusion separation model We present here a simple estimate of the resolution that can be achieved with our microfluidic diffusional separation device, neglecting the parabolic flow profile. This is a good approximation in the y-direction (parallel to the glass slide), but less accurate in the z-direction (perpendicular to the glass slide), as the channel is much wider than it is high. For species that do not diffuse far from the central region of the microfluidic channel, wall effects can be neglected. Assuming a plug flow in a microfluidic channel with initial conditions described by a delta function and a boundary condition of zero flow, the diffusion equation is $\frac{\partial \Phi}{\partial t}=D \frac{\partial^{2} \Phi}{\partial x^{2}}$, where $\phi$ is the concentration, $\mathrm{t}$ is time, $\mathrm{D}$ is the diffusion coefficient and $\mathrm{x}$ is the lateral position of the microfluidic channel. The kernel of the differential operator is $\Phi(x, t)=\frac{1}{2 \sqrt{\pi D t}} e^{-\frac{x^{2}}{4 D t}}$. The diffusion coefficient, $D$, can be expressed as a function of $\mathrm{r}_{H}$ (the hydrodynamic radius) and $\eta$ (the viscosity) as $D=\frac{k_{B} T}{6 \pi \eta r_{H}}$. The concentration ratio of species having two sizes $\left(\mathrm{r}_{H 1}, \mathrm{r}_{H 2}\right)$ and diffusion coefficients $\left(D_{1}, D_{2}\right)$ close to the channel centre $(x \approx 0)$ after one separation step is $\frac{\Phi_{1}}{\Phi_{2}}=\frac{\sqrt{D_{2}}}{\sqrt{D_{1}}}=\frac{\sqrt{r_{H 1}}}{\sqrt{r_{H 2}}}$. We can, therefore, achieve a purity of ca. $90 \%$ in the central channel after two separation steps for two types of particles with the ratio of diffusion coefficients equal to 10 . The nanostructures we use 
(protein fibrils and monomers) have a diffusion coefficient ratio around 50. The typical lengths of amyloid fibrils are of the order of hundred nanometres to several $\mu \mathrm{m}$, while the protein monomers are only several nanometres in diameter. In the experiments involving ThT binding to amyloid fibrils, the molecular mass of ThT molecules $(319 \mathrm{Da})$ is yet significantly smaller than that of protein monomers (several thousand Da). The fluorescence of samples collected from the central channel (originating from the specific binding of ThT to protein fibrils) was around 100k (a.u.) while only one hundred for those from the side channels (ThT only). Therefore, a size separation of $\leq 99 \%$ efficiency was achieved for insulin fibrils/ThT molecules in a single step. Furthermore, devices can be optimised and multi-step devices developed to enhance performances; indeed in principle any desired levels of purity can be achieved.

Analysis of binding equilibria The equilibrium between a ligand and a protein monomer or aggregate can be expressed as $\mathrm{P}+\mathrm{L} \rightleftharpoons \mathrm{PL}$, where $\mathrm{P}$ is the protein, $\mathrm{L}$ is the ligand and $\mathrm{PL}$ is the complex after binding. ${ }^{7}$ The dissociation constant $\mathrm{K}_{d}$ for this reaction is $K_{d}=\frac{[P][L]}{[P L]}$ where $[\mathrm{X}]$ denotes the concentration of species $\mathrm{X} . \mathrm{K}_{d}$ gives the concentration of free ligand at which half of the binding sites are occupied. The fractional saturation $\mathrm{Y}$ is defined as the concentration of binding ligand over the concentration of total protein: $Y=\frac{[P L]}{[P]+[P L]}$, which can be rearranged into $Y=\frac{[L]}{[L]+K_{d}}$, and which can be used to fit the experimental binding curve. In the presence of $\mathrm{n}$ independent binding sites per protein molecule, the equation is modified to yield $Y=\frac{n[L]}{[L]+K_{d}}$. In the case of amyloid fibrils, the experimentally determined stoichiometry is smaller than $1(\sim 0.1)$, indicating that within an amyloid fibril a total of approximately 10 protein molecules provide a binding site for one molecule of ThT, in good agreement to what has been reported previously for insulin amyloid fibrils under similar conditions. ${ }^{8}$

\section{References}

(1) Knowles, T. P. J.; Shu, W.; Devlin, G. L.; Meehan, S.; Auer, S.; Dobson, C. M.; Welland, M. E. Proc Natl Acad Sci U S A 2007, 104, 10016-10021. 
(2) Hoyer, W.; Antony, T.; Cherny, D.; Heim, G.; Jovin, T. M.; Subramaniam, V. J Mol Biol 2002, 322, 383-393.

(3) Pinotsi, D.; Buell, A. K.; Galvagnion, C.; Dobson, C. M.; Schierle, G. S. K.; Kaminski, C. F. Nano Lett 2014, 14, 339-345.

(4) Guilliams, T.; El-Turk, F.; Buell, A. K.; O’Day, E. M.; Aprile, F. A.; Esbjörner, E. K.; Vendruscolo, M.; Cremades, N.; Pardon, E.; Wyns, L.; Welland, M. E.; Steyaert, J.; Christodoulou, J.; Dobson, C. M.; Genst, E. D. J Mol Biol 2013, 425, 2397-2411.

(5) Duffy, D. C.; McDonald, J. C.; Schueller, O. J. A.; Whitesides, G. M. Anal. Chem. 1998, 70, 1998, 70, 4974-4984.

(6) Buell, A. K.; Galvagnion, C.; Gaspar, R.; Sparr, E.; Vendruscolo, M.; Knowles, T. P. J.; Linse, S.; Dobson, C. M. Proc Natl Acad Sci U S A 2014, 111(21), 7671-7676.

(7) Bolisetty, S.; Mezzenga, R. Nat Nanotechnol 2016, 11, 365-371.

(8) Groenning, M.; Norrman, M.; Flink, J. M.; van de Weert, M.; Bukrinsky, J. T.; Schluckebier, G.; Frokjaer, S. J Struct Biol 2007, 159, 483-497. 